\title{
Survival Rates for Porcelain Laminate Veneers: A Systematic Review
}

\author{
Yousra H. AlJazairy ${ }^{1}$ \\ ${ }^{1}$ Department of Restorative Dental Sciences, College of Dentistry, \\ King Saud University, Riyadh, Saudi Arabia
}

Eur J Dent 2021;15:360-368

\begin{abstract}
Address for correspondence Yousra Hussain AlJazairy, BDS, MSc, Department of Restorative Dental Sciences, College of Dentistry, King Saud University P.O. Box 60169, Riyadh 11426, Saudi Arabia (e-mail: yousra.aljazairy@gmail.com).
\end{abstract}

\author{
Abstract \\ Keywords \\ - porcelain laminate \\ veneers \\ - longevity \\ - follow-up \\ - failure \\ - ceramic \\ - restorations \\ - homogeneity
}

The aim of this systematic review was to analyze and compare the most up-to-date information available on long-term, medium-term, and short-term survival rates of porcelain laminate veneers (PLVs) and investigate the homogeneity in current studies or lack of it. An electronic search was performed using PubMed, Ovid MEDLINE, Cochrane Library, Web of Science, EBSCO, Science Direct, Wiley, and Scopus databases. Based on the PRISMA guidelines, the main inclusion criteria consisted of research articles published after the year 2000, in vivo studies with a follow-up period of at least 1 year and reporting of the Kaplan-Meier estimated cumulative survival rates. Quality assessment of the included studies was performed using the modified systematic assessment list consisting of 24 items. Thirty full-text articles were reviewed in detail. A total of 30 articles met the inclusion criteria and were selected for qualitative synthesis. The remaining 27 publications were retained to discuss the heterogeneity in the current literature and reported longevity of veneer restorations. A conclusive estimation of the longevity of PLVS beyond 20 years is lacking. The availability of evidence in the current literature is limited in terms of sample size and duration of follow-up. However, the majority of studies have concluded that PLVs have high-success rates and predictable patient outcomes. The present literature indicates an increased heterogeneity among research study designs. Researchers should aim for homogeneous study designs that can be included in systematic reviews and meta-analyses.

\section{Introduction}

In modern dentistry, porcelain veneer restorations (PVRs) have garnered a reputation as one of the most successful modalities of treatment. ${ }^{1}$ Porcelain restorations, specifically porcelain laminate veneers (PLVs), are popular among both dentists and patients due to their ability to replicate the lifelike appearance and luster of natural teeth.

The success of PLVs can be attributed to the ability of these veneer restorations to closely mimic the balanced relationship between biological, mechanical, functional, and esthetic parameters of natural teeth. ${ }^{2}$ Some of these parameters include predictable outcomes, superior esthetics, long-lasting color stability, life-like translucency, high-abrasion resistance,

published online

October 1, 2020
DOI https://doi.org/ 10.1055/s-0040-1715914 ISSN 1305-7456. outstanding resistance of fluid absorption, practical compressive, tensile and shear strengths, exceptional marginal integrity, biocompatibility with gingival tissue, greater conservation of tooth structure with minimal tooth reduction, and excellent long-term durability. ${ }^{1,3}$ The last two parameters are highly debatable topics among researchers.

Researchers have credited this success to a clinician's ability to properly, plan a case, select appropriate ceramics to use, select the materials and methods of cementation, conservative preparation of teeth, implement high-caliber finishing and polishing, and effectively plan for the continuing maintenance of the restorations. ${ }^{1}$ When applied correctly, these guidelines have been highly effective in remedying clinical defects such as correcting

\footnotetext{
(c) 2020. European Journal of Dentistry.

This is an open access article published by Thieme under the terms of the Creative Commons Attribution-NonDerivative-NonCommercial-License, permitting copying and reproduction so long as the original work is given appropriate credit. Contents may not be used for commercial purposes, or adapted, remixed, transformed or built upon. (https://creativecommons.org/licenses/by-nc-nd/4.0/)

Thieme Medical and Scientific Publishers Pvt. Ltd., A-12, 2nd Floor, Sector 2, Noida-201301 UP, India
} 
tooth forms and position, closing diastemata, replacing old composite restorations, restoring teeth with incisal abrasions or tooth erosion, masking enamel defects, and covering or reducing tooth discolorations such as fluorosis and tetracycline staining. ${ }^{4}$

Despite their successful reputation and similar to other alternative restorative treatments, PLVs are also prone to failure. Several clinical trials, systematic reviews, and meta-analyses have reported a wide range of survival rates over the past few decades. ${ }^{5-9}$ These reported survival rates, although extremely valuable, are still considered inconclusive or contradictory when viewed by the general consensus of the scientific community. For example, studies with a follow-up period of less than 5 years have reported survival rates ranging from 80.1 to $100 \%{ }^{10-17}$ Studies with a follow-up from 5 years up to 7 years have reported a range of 47 to $100 \% .^{18-28}$ Studies from 10 to 12 years have stated survival rates ranging from 53 to $94.4 \% .429-33$

Two of the longest studies conducted to conclude the survival rates of veneers are worth mentioning separately. Friedman in 1998 conducted the longest retrospective cohort study with a follow-up period of up to 15 years. ${ }^{34}$ The study reported a straight percentage outcome of $93 \%$ for 3,255 veneers. Layton \& Walton ${ }^{35}$ in 2007 reported the results of their prospective cohort with a Kaplan-Meier estimated cumulative survival rate of $73 \%$. The study included 100 patients treated with 304 veneers with a maximum follow-up period of up to 16 years. However, there is a lack of long-term studies and the wide range of inconsistent results can be observed in some of the studies conducted previously to evaluate the clinical success of PLVs. These varying results can be blamed on the overall heterogeneity of the study designs. More specifically, the conflicting results depend on several influencing factors. The definition of "failure" is the most basic of these factors. The main difference being, some researchers count a veneer as a failure only when it is "irreparable,", 41 while others mark a restoration a failure even if it is "reparable." ${ }^{29,30}$ The use of different evaluation criteria (California Dental Association (CDA)/Ryge, ${ }^{34}$ US Public Health Service (USPHS), ${ }^{23,24}$ FDI, ${ }^{10,15}$ Walton's Six Field $^{35}$ ) is another influencing factor. Other factors that make different reports hard to compare are reporting of survival rates in straight percentages ${ }^{19,26-28,34}$ or using a Kaplan-Meir analysis, ${ }^{29,30,35,36}$ taking into account or ignoring clustered outcomes $^{20,21}$, prospective ${ }^{12,14,16}$ versus retrospective ${ }^{17,29,30}$, direction of trials, and inconclusive or missing information. Finally, use of "modified" criteria (Modified CDA/Ryge,, $, 11,31$ Modified USPHS $12,13,18,19,25$ ) and methodologies (modified Kaplan-Meier ${ }^{35}$ ) have further increased the heterogeneity of the conducted studies.

Despite the heterogeneity of these studies, it can be safely stated that there are ample short- to medium-term studies with high-success rates. However, according to some researchers, the survival rate of PLVs should be no less than $100 \%$, especially for short-term studies. ${ }^{8}$ To assess the true longevity of the PLVs, it is perhaps acceptable to describe the overall clinical success of PLVs on the basis of short-, medium-, and long-term definitions. Therefore, this review has focused on investigating studies with a follow-up period of a minimum of 1 year up to more than 20 years. The aim of this systematic review was to analyze and compare the most up-to-date information available on short-, medium-, and long-term survival rates of PLVs and investigate the homogeneity in current studies or lack of it.

\section{Materials and Methods}

\section{Standard of Reporting and PICOS Principle}

The present systematic review followed the preferred reporting items for systematic reviews and meta-analysis (PRISMA) guideline. The studies were identified using the following PICOS principle: Patients $=$ patients who received ceramic veneers, Intervention $=$ PLVs, Control (not applicable in the present study), Outcome = estimation of Kaplan-Meier cumulative survival rate and Study design = prospective or retrospective studies.

\section{Study Selection}

The main inclusion criteria consisted of articles published in the English language, foreign language articles with readily available translations, in vivo studies with a follow-up of at least 1 year, both retrospective and prospective randomized controlled trials (RCTs) in humans, and reporting of a KaplanMeier estimated cumulative survival rate. Studies were not considered if studies did not report adequate description related to the preparation of PLVs or their bonding procedures, review articles, case reports, abstracts or unpublished data.

\section{Data Sources}

An extensive electronic search was performed using PubMed, Ovid MEDLINE, Cochrane Library, Web of Science, EBSCO, Science Direct, Wiley, and Scopus databases from 1970 to 2020. The search was conducted using different combinations of the following terms: "dental," "porcelain," "ceramic," "laminate," "veneers," "PLVs" "survival," “rate," "longevity," "follow-up," "failure," "clinical," "performance," "retrospective," "prospective," and "longitudinal."

No manual or hand search was conducted. During the initial screening process, all duplicates were identified and removed. Other exclusions included research abstracts, posters, case reports, commentaries, critical appraisals, letters to editors, editorials, conference papers, and review articles. The references of all full-text articles including systematic reviews and meta-analyses were inspected for additional relevant sources. Unpublished studies (gray literature) were identified by searching the Open-GRAY database, and references of the included studies (cross referencing) were performed to obtain new studies. One reviewer (YHA) screened the titles and the abstracts of all the results identified through the electronic searches. Full articles were collected for titles requiring detailed inspection.

\section{Quality Assessment}

Quality of all the studies were assessed using a modified systematic assessment list consisting of 24 items. ${ }^{37}$ The 24 -item list was developed according to the guidelines of publication. 
The items consisted of points including the reporting of hypothesis, aims, setting/study design, distribution of study population by age and gender, adequate eligibility criteria, description of treatment, sample size, main outcomes, use of control group, randomization and blinding, calibration performed, adequate statistical analyses, reporting of confidence intervals, adverse effects, and conclusions. For each item, a single mark " $x$ " was given. A percentage value of quality items was analyzed for individual study.

\section{Results}

\section{Study Selection}

Out of the total 120 full-text articles, 65 articles were excluded for various reasons including in vitro studies, extracted teeth, CAD/CAM, materials unclear or not ceramic/porcelain, no Kaplan-Meier analysis, missing or unclear data, and missing or unclear conclusions. Another 25 publications were removed, as they were systematic reviews or meta-analyses. The remaining 30 full-text articles were reviewed in depth and used to discuss the heterogeneity in current literature and reported longevity of veneer restorations. The corresponding steps for initial screening and selection of studies are shown in the PRISMA diagram (-Fig. 1).

\section{General Description of the Included Studies}

The general characteristics of the selected studies are reported in - Table $\mathbf{1}$. Out of all the studies included, a total of
16 studies were retrospective, while a total of 14 studies were prospective cohort studies. On the basis of follow-up period, nine clinical trials had long-term follow up period ranging from 10.5 years to 50 years. Eight studies had medium-term follow-up ranging from 6 years to 10 years, while 13 shortterm studies reported a follow-up period of $<6$ years. The included studies initiated from the year 1966 to the year 2016. A total of 2473 patients were included in the included clinical trials. The total number of PLVs studied in the clinical trials were 11,465 .

\section{Main Outcomes of the Studies}

\section{Long-term Clinical Trials}

All long-term clinical trials reported their outcomes based on the Kaplan-Meier analysis. The criteria used for assessing survival were variable. Three studies were author-defined, ${ }^{29,38,39}$ two studies used Walton's Six Field ${ }^{35,36}$ and modified CDA/ Ryge, ${ }^{4,31}$ while only one study used CDA/Ryge. ${ }^{34}$ The overall survival rate ranged from $100 \%$ to $73 \%$ in the included longterm trials. A general trend of reduced survival rate was observed among four studies who reported their survival rates at different time points. ${ }^{29,31,35,39}$

\section{Medium-term Clinical Trials}

Four clinical trials reported the survival outcomes based on the Kaplan-Meier analysis. ${ }^{18,22,23,32}$ One study reported mean percentage of survival rate, ${ }^{19}$ while outcomes from the three studies were either unclear or had missing data. ${ }^{20,21,33}$

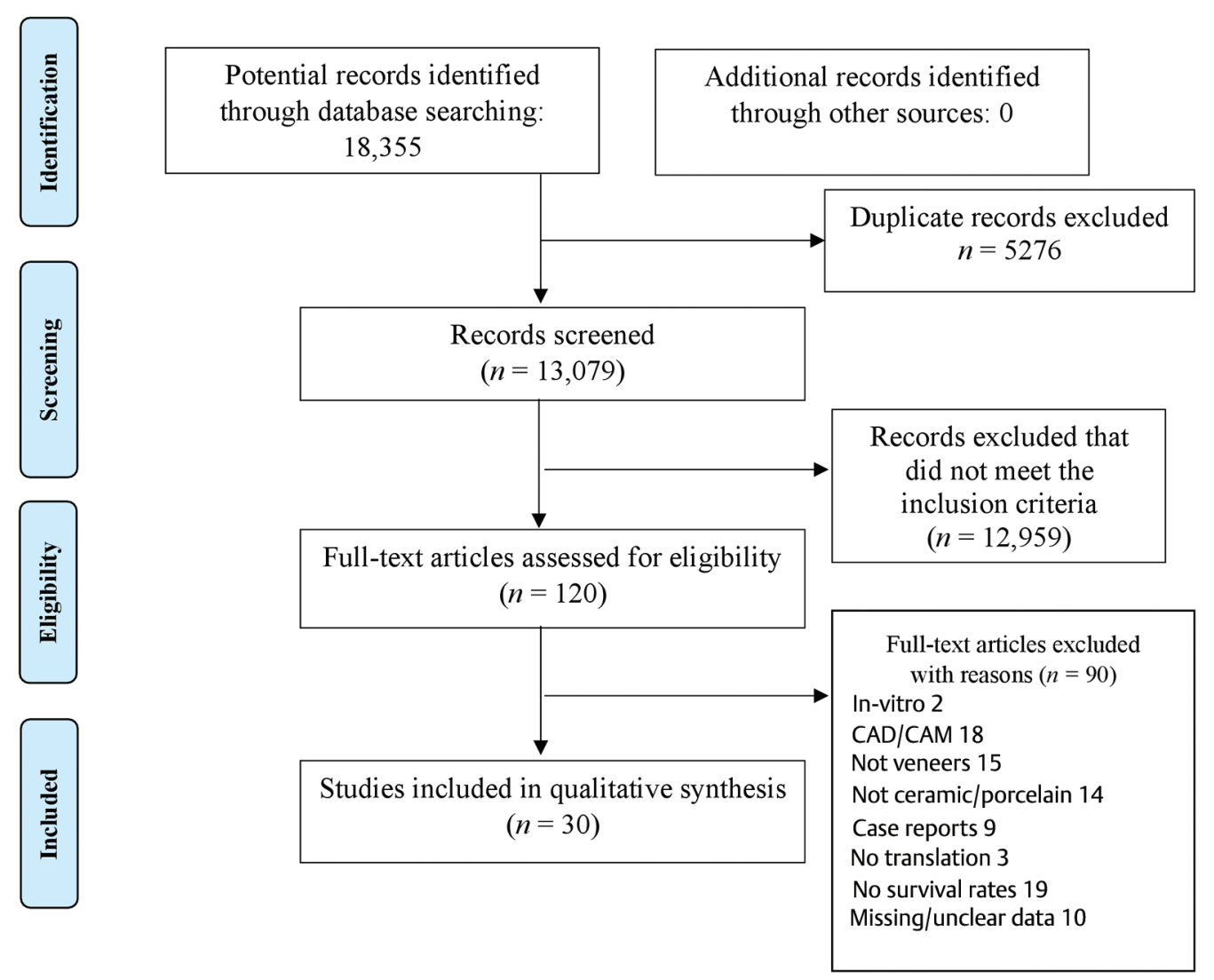

Fig. 1 PRISMA flowchart for the study selection process. 
Table 1 Characteristics of selected articles

\begin{tabular}{|c|c|c|c|c|c|c|c|c|c|}
\hline$\#$ & Author & Period & $\begin{array}{l}\text { Max. } \\
\text { follow-up }\end{array}$ & Patients & PLVs & Survival rate & Criteria & $\begin{array}{l}\text { Survival } \\
\text { method }\end{array}$ & $\begin{array}{l}\text { Study } \\
\text { design }\end{array}$ \\
\hline \multicolumn{10}{|c|}{ Long-term trials } \\
\hline 1 & Olley et al $(2018)^{38}$ & 1966-2016 & 50 years & * & 22 & $100 \%$ & A & KM & $\mathrm{RC}$ \\
\hline 2 & Layton \& Walton (2012) ${ }^{36}$ & $1990-2010$ & 21 years & 155 & 499 & $96 \%$ & W & KM & PC \\
\hline 3 & Beier et al $(2012)^{39}$ & 1987-2009 & 20 years & 84 & 318 & $\begin{array}{l}94.4 \%-5 \text { years } \\
93.5 \%-10 \text { years } \\
82.93 \%-20 \text { years }\end{array}$ & A & $\mathrm{KM}$ & $\mathrm{RC}$ \\
\hline 4 & Layton \& Walton (2007) ${ }^{35}$ & $1988-2003$ & 16 years & 100 & 304 & $\begin{array}{l}96 \%-5 \text { to } \\
6 \text { years } 93 \%-10 \text { to } \\
11 \text { years } 91 \%-12 \text { to } \\
13 \text { years } 73 \%-15 \text { to } \\
16 \text { years }\end{array}$ & W & KM & PC \\
\hline 5 & Friedman $(1998)^{34}$ & $*$ & 15 years & $*$ & 3500 & $93 \%$ & C & $\%$ & $\mathrm{RC}$ \\
\hline 6 & Gurel et al $(2013)^{29}$ & 1997-2009 & 12 years & 66 & 580 & $\begin{array}{l}92 \%-6 \text { years } 86 \%- \\
12 \text { years }\end{array}$ & A & KM & $\mathrm{RC}$ \\
\hline 7 & Fradeani et al $(2005)^{4}$ & 1991-2002 & 12 years & 46 & 182 & $94.40 \%$ & $\mathrm{MC}$ & KM & $\mathrm{RC}$ \\
\hline 8 & Granell-Ruiz et al (2009) ${ }^{30}$ & 1995-2003 & 11 years & 70 & 323 & $\begin{array}{l}\text { 94\%-simple } 85 \%- \\
\text { functional }\end{array}$ & * & KM & $\mathrm{RC}$ \\
\hline 9 & $\begin{array}{l}\text { Dumfahrt \& Schäffer } \\
(2000)^{31}\end{array}$ & 1986-1997 & 10.5 years & 65 & 191 & $\begin{array}{l}97 \%-5 \text { years } 91 \%- \\
10.5 \text { years }\end{array}$ & $\mathrm{MC}$ & KM & $\mathrm{RC}$ \\
\hline \multicolumn{10}{|c|}{ Medium-term trials } \\
\hline 10 & Burke \& Lucarotti (2009) 32 & 1991-2001 & 10 years & 1177 & 2562 & $53 \%$ & $*$ & KM & $\mathrm{RC}$ \\
\hline 11 & Peumans et al $(2004)^{33}$ & 1990-2000 & 10 years & 25 & 87 & $64 \%$ & A & $*$ & PC \\
\hline 12 & D’Arcangelo et al (2012) ${ }^{18}$ & 2002-2008 & 7 years & 30 & 119 & $97.50 \%$ & $\mathrm{MU}$ & KM & PC \\
\hline 13 & Shao-Ping et al $(2012)^{19}$ & 2005-2012 & 7 years & 32 & 206 & $97.60 \%$ & $\mathrm{MU}$ & $\%$ & $\mathrm{RC}$ \\
\hline 14 & Smales \& Etemadi $(2004)^{20}$ & 1993-2000 & 7 years & 50 & 110 & $\begin{array}{l}\text { 95.8\%-incisal } \\
\text { 85.5\%-nonincisal }\end{array}$ & $*$ & $*$ & $\mathrm{RC}$ \\
\hline 15 & Magne et al $(2000)^{21}$ & 1995-1999 & 7 years & 16 & 48 & $100 \%$ & A & $*$ & $\mathrm{RC}$ \\
\hline 16 & Shaini et al $(1997)^{22}$ & 1984-1992 & 6.5 years & 102 & 372 & $47 \%$ & A & KM & $\mathrm{RC}$ \\
\hline 17 & Fradeani $(1998)^{23}$ & 1991-1997 & 6 years & 21 & 83 & $99 \%$ & $\mathrm{U}$ & KM & PC \\
\hline \multicolumn{10}{|c|}{ Short-term trials } \\
\hline 18 & Aykor \& Ozel $(2009)^{24}$ & 1991-1997 & 5 years & 30 & 300 & $94-95 \% *$ & $U$ & $*$ & PC \\
\hline 19 & Guess \& Stappert $(2008)^{25}$ & 1999-2006 & 5 years & 25 & 66 & $\begin{array}{l}\text { 100\%-full } \\
97.5 \% \text {-overlap }\end{array}$ & $\mathrm{MU}$ & KM & PC \\
\hline 20 & Murphy et al $(2005)^{26}$ & 1996-2001 & 5 years & 29 & 62 & $89 \%$ & * & $\%$ & $\mathrm{RC}$ \\
\hline 21 & $\begin{array}{l}\text { Aristidis \& Dimitra } \\
(2002)^{27}\end{array}$ & 1993-1998 & 5 years & 61 & 186 & $98.40 \%$ & A & $\%$ & PC \\
\hline 22 & Peumans et al $(1998)^{28}$ & 1990-1995 & 5 years & 25 & 87 & $93 \%$ & A & $\%$ & PC \\
\hline 23 & $\begin{array}{l}\text { Coelho-de-Souza et al } \\
(2015)^{10}\end{array}$ & $*$ & 3.5 years & 86 & 196 & $80.10 \%$ & $\mathrm{~F}$ & KM & $\mathrm{RC}$ \\
\hline 24 & Fabbri et al (2014) & 2006-2010 & 3.5 years & $*$ & 318 & $97.91 \%$ & $\mathrm{MC}$ & KM & PC \\
\hline 25 & Gresnigt et al $(2013)^{12}$ & 2007-2010 & 3.3 years & 20 & 92 & $94.60 \%$ & $\mathrm{MU}$ & KM & PC \\
\hline 26 & Rinke et al (2013) ${ }^{13}$ & 2008-2010 & 3 years & 37 & 130 & $95.10 \%$ & $\mathrm{MU}$ & KM & $\mathrm{RC}$ \\
\hline 27 & Nordbø et al (1994) ${ }^{14}$ & 1990-1993 & 3 years & 41 & 135 & $98.50 \%$ & $*$ & $*$ & PC \\
\hline 28 & $\begin{array}{l}\text { Karagözoğlu et. al. } \\
(2016)^{15}\end{array}$ & $*$ & 2 years & 12 & 62 & $100 \%$ & $\mathrm{~F}$ & KM & PC \\
\hline 29 & Öztürk \& Bolay $(2014)^{16}$ & 2008-2011 & 2 years & 28 & 125 & $91.20 \%$ & $\mathrm{MU}$ & KM & PC \\
\hline 30 & Çötert et al (2009) ${ }^{17}$ & 1999-2005 & 1.5 years & 40 & 200 & $99.50 \%$ & A & KM & $\mathrm{RC}$ \\
\hline
\end{tabular}

Abbreviations: \%, straight percentage; A, author-defined; C, CDA/Ryge, F, FDI; KM, Kaplan-Meier analysis; MC, modified CDA/Ryge; MU, modified USPHS; P, prospective cohort; $R$, retrospective cohort; U, USPHS; W, Walton's six field.

$\left(^{*}\right)$ Unclear or missing data.

Three studies used their own criteria, ${ }^{21,22,33}$ two studies used modified USPHS, ${ }^{18,19}$ while one study used USPHS criteria. ${ }^{23}$ The overall survival rate ranged from $100 \%$ to $47 \%$ in medium-term clinical trials.

\section{Short-term Clinical Trials}

A total of eight trials were of short-term, which estimated the survival rate of PLVs using the Kaplan-Meier analysis. ${ }^{10-13,15-17,25}$ Three trials reported the survival rate using 
mean percentage, ${ }^{26-28}$ four studies used the modified USPHS criteria, ${ }^{12,13,16,25}$ three studies using author-defined, ${ }^{17,27,28}$ two studies using FDI, ${ }^{10,15}$ while one study each using modified CDA/Ryge ${ }^{11}$ and USPHS. ${ }^{24}$

\section{Quality Assessment}

The majority of studies achieved $>75 \%$ on the quality assessment. The percentage range of the trials selected in the quality estimation ranged from 58\% to 96\% (-Table 2).

\section{Discussion}

Systematic reviews and meta-analyses are the core of evidence-based dentistry. ${ }^{40}$ Clinical trials are, in turn, critically fundamental in supporting the quality of evidence synthesis for both systematic reviews and meta-analyses. ${ }^{41}$ The homogeneity of studies is therefore of utmost importance in conducting beneficial systematic reviews. Researchers have suggested that reporting in systematic reviews can be improved by universally agreed upon standards and guidelines. ${ }^{42}$ For instance, it is worth noting that previously in 2007, Hickel et $\mathrm{al}^{43}$ have called for evidence-based studies to follow homogeneous study designs in order for future RCTs to be subsequently included in systematic reviews and meta-analyses. In their publication, the authors promoted the use of the FDI criteria and laid out a detailed framework for researchers to follow in designing and conducting their research.

Carrying out RCTs with large sample sizes and over lengthy follow-up periods are often difficult. As an alternative, well-designed systematic reviews can provide reliable answers to research questions by analyzing several RCTs. ${ }^{44}$ For example, the questions of longevity of PLVs and precisely predicting their treatment outcomes. On the other hand, researchers find it difficult to combine RCTs with incomparable statistical variables.

The studies examined in this literature review (- Table 1) clearly demonstrated that studies with contradistinctive reporting factors are difficult to combine usefully for systematic reviews or meta-analyses. Without inspecting the detailed methods and materials, and only observing three basic factors such as definition of failure, evaluation criteria and statistical methodology, a wide range of disparity can be noticed.

Out of the total 27 studies, 13 studies were designed as prospective clinical trials and 14 studies were retrospective cohorts. Failure was defined as "irreparable" in 13 studies, ${ }^{4,11-13,15,18,22,23,25,27,31,34,35}$ and "reparable but counted as a failure" in nine studies. . $^{10,14,16,17,28-30,32,33}$ The remaining five studies either did not state or unclearly stated the definition of failure. ${ }^{19,20,21,24,26}$ In terms of evaluation criteria, seven studies ${ }^{17,21,22,27-29,33}$ used an author-defined criteria, six studies ${ }^{12,13,16,18,19,25}$ used the modified USPHS criteria, three studies ${ }^{4,11,31}$ used the modified CDA/Ryge criteria, two studies $^{10,15}$ used the FDI criteria and another two ${ }^{23,24}$ used the USPHS criteria, one study ${ }^{34}$ each used the CDA/Ryge criteria and ${ }^{35}$ the Walton's Six Field criteria, while five studies $^{14,20,26,30,32}$ did not report a clear criteria. In utilizing proper survival estimation methodology, five studies ${ }^{19,26-28,34}$ used straight percentages, and another five studies reported

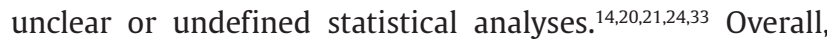
17 studies, being a definite majority, did utilize the KaplanMeier analysis and reported a precise cumulative survival estimation. ${ }^{4,10-13,15-18,22,23,25,29-32,35}$

At the very basic, if the definitions of failure were standardized, the survival times would be the same conceptually. If the studies used the Kaplan-Meier analysis instead of straight percentages, then the statistical results can be aggregated into information, leading to a higher statistical power and conclusions that are more robust. Finally, using the same evaluation criteria could aid in decreasing the researcher bias in judging the state of a restoration and could provide results that could be compared in depth, according to a detailed breakdown of esthetic, functional, and biological properties.

The same can be observed for the three studies retained for qualitative synthesis. All the authors defined their own evaluation criteria. Even though Walton's Six Field classification has been standardized, it has not been employed in any other study. The only use that can be seen in the current literature is in studies conducted by the same authors. ${ }^{7,35,36}$ Survival rate estimations were calculated using the KaplanMeier analysis by all three of the studies. However, Layton \& Walton ${ }^{36}$ used a slightly modified version of the analysis, and Olley et $\mathrm{al}^{38}$ did not state clearly whether the methodology was used to calculate the survival rate of the veneers or only the crowns, rather defined failure in detail but ambiguously, while the remaining two studies defined failure as an irreparable problem. The specifics of the three studies can be observed in more detail below.

Beier et $\mathrm{al}^{39}$ in their retrospective study conducted a longterm analysis of up to 20 years. The study was conducted at the Innsbruck Medical University in Innsbruck, Austria. Two associate professors placed 318 silicate glass ceramic veneers in 84 patients ( 38 males, 46 females). The study population consisted of 42 patients (50\%) with bruxism and 23 smokers (27.28\%). The restorations were placed between November 1987 and December 2009. The fabrication of PLVs varied according to the placement period and included feldspathic porcelain, leucite heat pressed ceramic, or lithium disilicate heat-pressed ceramic. The veneers were evaluated between March 2010 and July 2010 by using the modified CDA/Ryge criteria. In addition, a papilla bleeding index (PBI) assessment and a customer satisfaction survey were conducted. Out of the total 318 veneers, 152 veneers were observed over 10 years, 75 veneers were observed over 17 years, and only three veneers were observed over 20 years.

Failure was defined as an "irreparable problem.” Twenty veneers failed before the evaluation in 2010 and no clinical data using modified CDA/Ryge was recorded. However, since the type of failure was recorded, the veneers were not censored from the Kaplan-Meier analysis. The authors did provide a breakdown of failures according to CDA/Ryge criteria 
Table 2 Quality assessment of the included studies

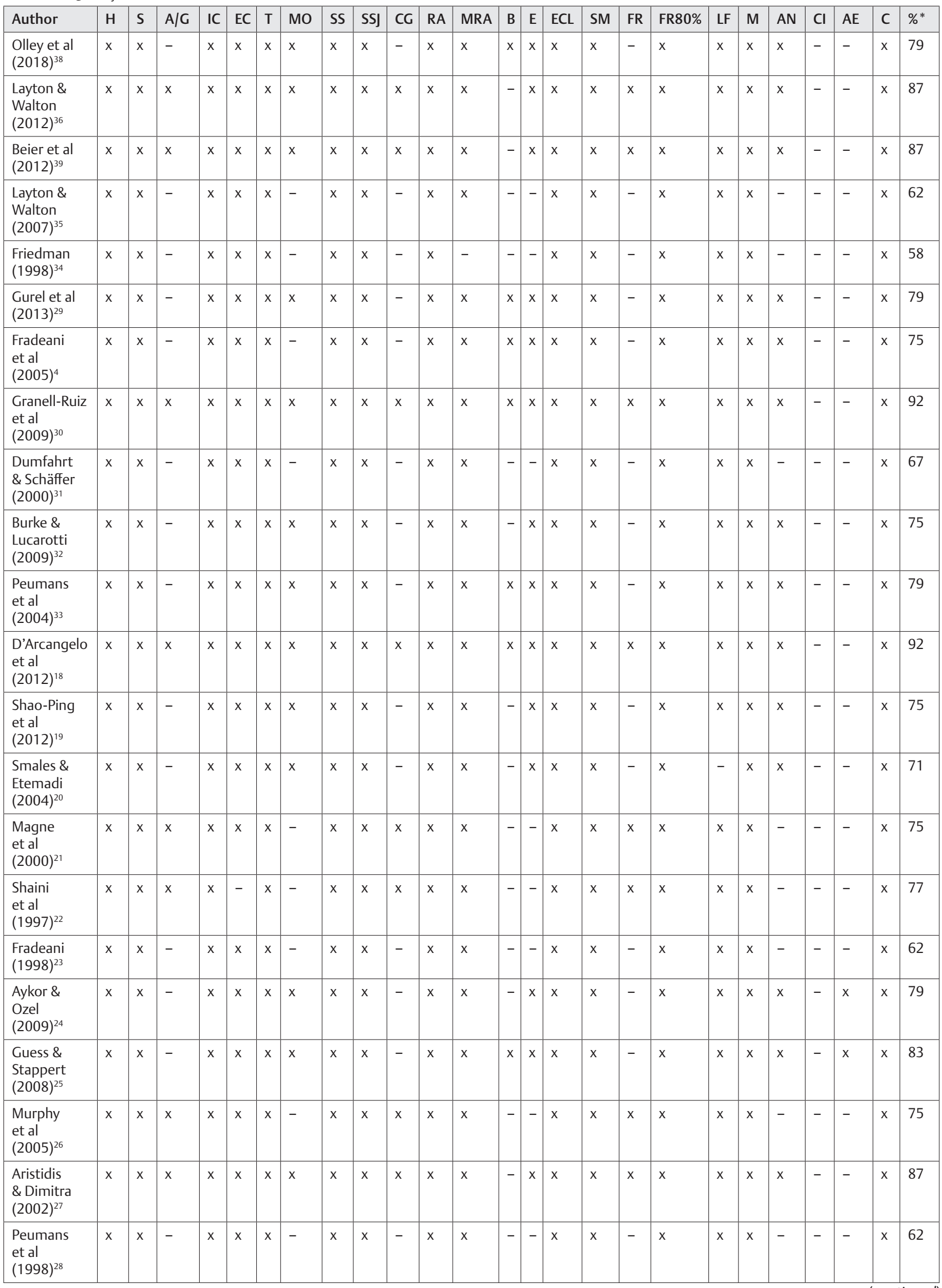


Table 2 (continued)

\begin{tabular}{|c|c|c|c|c|c|c|c|c|c|c|c|c|c|c|c|c|c|c|c|c|c|c|c|c|c|}
\hline Author & $\mathrm{H}$ & $S$ & $A / G$ & IC & EC & $\mathrm{T}$ & MO & SS & SS] & CG & RA & MRA & B & $\mathrm{E}$ & $\mathrm{ECL}$ & SM & FR & FR80\% & LF & $M$ & AN & $\mathrm{Cl}$ & $\mathrm{AE}$ & C & $\% *$ \\
\hline $\begin{array}{l}\text { Coelho- } \\
\text { de-Souza } \\
\text { et al } \\
(2015)^{10}\end{array}$ & $x$ & $x$ & $x$ & $x$ & $x$ & $x$ & - & $x$ & $x$ & $x$ & $x$ & $x$ & $x$ & - & $x$ & $x$ & $x$ & $x$ & $x$ & $x$ & - & - & - & $x$ & 79 \\
\hline $\begin{array}{l}\text { Fabbri et al } \\
(2014)^{11}\end{array}$ & $x$ & $x$ & $x$ & - & $x$ & $x$ & - & $x$ & $x$ & $x$ & $x$ & $x$ & - & - & $x$ & $x$ & $x$ & $x$ & $x$ & $x$ & - & - & $x$ & $x$ & 75 \\
\hline $\begin{array}{l}\text { Gresnigt et } \\
\text { al }(2013)^{12}\end{array}$ & $x$ & $x$ & $x$ & $x$ & $x$ & $x$ & - & $x$ & $x$ & $x$ & $x$ & $x$ & $\mathrm{x}$ & - & $x$ & $x$ & $x$ & $x$ & $x$ & $x$ & - & - & - & $x$ & 79 \\
\hline $\begin{array}{l}\text { Rinke et al } \\
(2013)^{13}\end{array}$ & $x$ & $x$ & $x$ & $x$ & $x$ & $x$ & $x$ & $x$ & $x$ & $x$ & $x$ & $x$ & - & $\mathrm{x}$ & $x$ & $x$ & $x$ & $x$ & $x$ & $x$ & $x$ & - & - & $x$ & 87 \\
\hline $\begin{array}{l}\text { Nordbø et al } \\
(1994)^{14}\end{array}$ & $x$ & $x$ & - & $x$ & $x$ & $x$ & $x$ & $x$ & $x$ & - & $x$ & $x$ & - & $\mathrm{x}$ & $x$ & $x$ & - & $x$ & $x$ & $x$ & $x$ & - & - & $x$ & 75 \\
\hline $\begin{array}{l}\text { Karagözoğlu } \\
\text { et. al. } \\
(2016)^{15}\end{array}$ & $x$ & $x$ & $x$ & $x$ & $x$ & $x$ & $x$ & $x$ & $x$ & $x$ & $x$ & $x$ & $\mathrm{x}$ & $\mathrm{x}$ & $x$ & $x$ & $x$ & $x$ & $x$ & $x$ & $x$ & - & $x$ & $x$ & 96 \\
\hline $\begin{array}{l}\text { Öztürk } \\
\text { \& Bolay } \\
(2014)^{16}\end{array}$ & $x$ & $x$ & $x$ & $x$ & $x$ & $x$ & $x$ & $x$ & $x$ & $x$ & $x$ & $x$ & $\mathrm{x}$ & $\mathrm{x}$ & $x$ & $x$ & $x$ & $x$ & $x$ & $x$ & $x$ & - & - & $x$ & 83 \\
\hline $\begin{array}{l}\text { Çötert et al } \\
(2009)^{17}\end{array}$ & $x$ & $x$ & $x$ & $x$ & $x$ & $x$ & $x$ & $x$ & $x$ & $x$ & $x$ & $x$ & - & $\mathrm{x}$ & $x$ & $x$ & $x$ & $x$ & $x$ & $x$ & $x$ & - & - & $x$ & 87 \\
\hline
\end{tabular}

Abbreviations: A/G, age/gender distribution described; AE, adverse events stated; AN, results stated in absolute numbers; B, blinding performed; C, conclusions stated; $\mathrm{CG}$, use of control group; $\mathrm{Cl}$, confidence intervals stated; $\mathrm{E}$, more than one examiner for outcome assessment; $\mathrm{EC}$, exclusion criteria; ECL, examiner calibration; FR, follow-up rate mentioned; FR80\%, follow-up rate greater than 80\%; H, hypothesis/aim described; IC, inclusion criteria; LF, lost to follow-up; M, main outcomes clearly described; MO, main outcomes to be measured; MRA, method of random allocation described; RA, random allocation to treatment used; S, study setting described; SM, statistical methods described; SS, sample size; SSJ, sample size justified; T, treatment described; $x$, yes.

$\%$ of questions answered yes.

by percentages, and the most frequent reason for failure was fracture of the ceramic (44.83\%).

The study also reported a significantly higher marginal discoloration among smokers and a significantly higher failure rate among bruxers. In total, approximately $12 \%$ of veneers were cemented without dentine bonding, and the authors attributed this to some of the restoration failures. Kaplan-Meier cumulative survival rates were reported for 5 years (94.4\%), 8 years (94.1\%), 10 years (93.5\%), 15 years (85.74\%) and 20 years (82.93\%).

The authors used the Cox proportional hazards model to study influence of various risk factors for failures. Instead of accounting for clustering, the authors computed robust standard errors by estimating a correlation between the observations from the same patient using methods described by Lin and Wei. ${ }^{45}$

Layton \& Walton ${ }^{36}$ in their prospective cohort study analyzed the survival rates of feldspathic porcelain veneers with a follow-up of 21 years. The restorations were placed in a private practice in Australia, by a single prosthodontist, between 1990 and 2010. A total of 499 veneers were placed in 155 patients. Patients with extensive loss of tooth structure through parafunction and unfavorable periodontal prognosis were excluded. Feldspathic porcelain veneers from refractory dies were etched, silanated and bonded. Only teeth with at least $80 \%$ enamel remaining were veneered. A total of 499 veneers were observed for the first 5 years, 354 veneers were observed up to 10 years, 239 veneers were observed up to 15 years, 82 veneers were observed up to 20 years, and only five veneers were observed for 21 years. The veneers were evaluated using the Walton's Six Field criteria and grouped into 5-year intervals. A total of 145 veneers were in situ for 1 to 5 years, 115 veneers were in situ from 10 to 15 years, 157 veneers were in situ for 10 to 15 years, 77 veneers were in situ from 15 to 20 years, and only five veneers survived more than 21 years.

Failure was defined as, when part or all of the prosthesis was lost, when marginal integrity was compromised, or when the veneer fell off more than twice. Walton's Six Field classification designates an outcome as "repair," when a veneer needs repair without interfering with the original marginal integrity of the restoration. Thus, it can be assumed that "failure" was defined as an irreparable problem. Eleven patients with 56 veneers experienced more than one outcome from Walton's criteria.

Kaplan-Meier survival rates were analyzed twice. First, for the entire sample of 499 veneers without accounting for clustering and then the outcome was analyzed for one randomly chosen veneer from each patient. This study was one of the very few studies that have emphasized and analyzed a survival rate while accounting for clustered outcomes. For the entire sample, Kaplan-Meier cumulative survival rates were reported for 5 years (98\%), 10 years (96\%), 15 years (91\%), and 20 years (91\%). For the randomly selected subsample, survival rates were reported as 5 years (98\%), 10 years (96\%), 15 years (96\%), and 20 years (96\%). The authors reported that survival rates were not significantly different for both groups.

Olley et al in their retrospective study investigated the outcome of indirect restorations with a follow-up of up to 50 years. ${ }^{38}$ The study was conducted at a mixed National 
Health Service (NHS)/private dental practice in London, United Kingdom. One operator placed 223 restorations in 47 patients between 1966 and 1996. The restorations consisted of metal-ceramic crowns (154), gold crowns (25), ceramic crowns (22), and ceramic veneers (22). However, only the 22 ceramic veneers placed in 10 patients are of importance for this review.

Only patients with excellent oral hygiene and favorable periodontal prognosis were included in this study. The only material detail given was that the restorations were feldspathic porcelain laminate veneers. Failure was defined as "issues that affected the survival of the restoration." Failures also included pulp infections or other periapical complications. The authors did not identify if a standard evaluation criterion was used, nor did they clearly state their own evaluation criteria. The Kaplan-Meier analysis was performed on all restorations, and the authors stated $100 \%$ veneers had survived at 50 years. However, it was unclear if the KaplanMeier estimated cumulative survival rate for the 22 veneers was $100 \%$ or if it was stated as a straight percentage.

The most critical observation that was made in all three of the studies was the small number of restorations evaluated beyond 20 years. It is evident that even though PLVs might have a longevity of 20 to 50 years, the dropout rate in RCTs over these periods is exceedingly high, and the outcomes reported represent a significantly small sample size. Although inconclusive in exact percentages, the majority of studies have concluded that porcelain laminate veneers have high-success rates and predictable patient outcomes.

\section{Conclusion}

A conclusive estimation of the longevity of porcelain laminate veneers beyond 20 years is lacking. The availability of evidence in the current literature is limited in terms of sample size and duration of follow-up. The present literature indicates an increased heterogeneity among research study designs. Researchers should aim for homogeneous study designs that can be included in systematic reviews and meta-analyses.

\section{Conflict of Interest}

None declared.

\section{Acknowledgement}

The author wish to thank the College of Dentistry Research Center at King Saud University, Saudi Arabia, for supporting this research project (project no. \#FR 0478).

\section{References}

1 Sá TCM, de Carvalho MFF, de Sá JCM, Magalhães CS, Moreira AN, Yamauti M. Esthetic rehabilitation of anterior teeth with different thicknesses of porcelain laminate veneers: An 8-year follow-up clinical evaluation. Eur J Dent 2018;12(4):590-593

2 Malchiodi L, Zotti F, Moro T. De Santis D, Albanese M. Clinical and esthetical evaluation of 79 lithium disilicate multilayered anterior veneers with a medium follow-up of 3 years. Eur J Dent 2019;13(4):581-588
3 Beltrami R, Ceci M, De Pani G, et al. Effect of different surface finishing/polishing procedures on color stability of esthetic restorative materials: a spectrophotometric evaluation. Eur J Dent 2018;12(01):049-56

4 Fradeani M, Redemagni M, Corrado M. Porcelain laminate veneers: 6- to 12-year clinical evaluation-a retrospective study. Int J Periodontics Restorative Dent 2005;25(1):9-17

5 Morimoto S, Albanesi RB, Sesma N, Agra CM, Braga MM. Main clinical outcomes of feldspathic porcelain and glass-ceramic laminate veneers: a systematic review and meta-analysis of survival and complication rates. Int J Prosthodont 2016;29(1):38-49

6 De, FA, da, Costa, G, Borges, BCD, de, Assunção, IV. . Clinical performance of porcelain laminate veneers with minimal preparation: a systematic review. Int J Experiment Dent Sci 2016;5(1):56-59

7 Layton DM, Clarke M, Walton TR. A systematic review and meta-analysis of the survival of feldspathic porcelain veneers over 5 and 10 years. Int J Prosthodont 2012;25(6):590-603

8 Burke FJ. Survival rates for porcelain laminate veneers with special reference to the effect of preparation in dentin: a literature review. J Esthet Restor Dent 2012;24(4):257-265

9 Kreulen CM, Creugers NHJ, Meijering AC. Meta-analysis of anterior veneer restorations in clinical studies. J Dent 1998;26(4):345-353

10 Coelho-de-Souza FH, Gonçalves DS, Sales MP, et al. Direct anterior composite veneers in vital and non-vital teeth: a retrospective clinical evaluation. J Dent 2015;43(11): 1330-1336

11 Fabbri G, Zarone F, Dellificorelli G, et al. Clinical evaluation of 860 anterior and posterior lithium disilicate restorations: retrospective study with a mean follow-up of 3 years and a maximum observational period of 6 years. Int J Periodontics Restorative Dent 2014;34(2):165-177

12 Gresnigt MM, Kalk W, Ozcan M. Randomized clinical trial of indirect resin composite and ceramic veneers: up to 3-year follow-up. J Adhes Dent 2013;15(2):181-190

13 Rinke S, Lange K, Ziebolz D. Retrospective study of extensive heat-pressed ceramic veneers after 36 months. J Esthet Restor Dent 2013;25(1):42-52

14 Nordbø H, Rygh-Thoresen N, Henaug T. Clinical performance of porcelain laminate veneers without incisal overlapping: 3-year results. J Dent 1994;22(6):342-345

15 Karagözoğlu İ, Toksavul S, Toman M. 3D quantification of clinical marginal and internal gap of porcelain laminate veneers with minimal and without tooth preparation and 2-year clinical evaluation. Quintessence Int 2016;47(6):461-471

16 Oztürk E, Bolay S. Survival of porcelain laminate veneers with different degrees of dentin exposure: 2-year clinical results. J Adhes Dent 2014;16(5):481-489

17 Cötert HS, Dündar M, Oztürk B. The effect of various preparation designs on the survival of porcelain laminate veneers. J Adhes Dent 2009;11(5):405-411

18 D'Arcangelo C, De Angelis F, Vadini M, D'Amario M. Clinical evaluation on porcelain laminate veneers bonded with lightcured composite: results up to 7 years. Clin Oral Investig 2012;16(4):1071-1079

19 Xu SP, Luo XP, Shi YJ. [Esthetic restoration for anterior teeth with the hot pressed porcelain laminate veneers]. Shanghai Kou Qiang Yi Xue 2012;21(5):572-575

20 Smales RJ, Etemadi S. Long-term survival of porcelain laminate veneers using two preparation designs: a retrospective study. Int J Prosthodont 2004;17(3):323-326

21 Magne P, Perroud R, Hodges JS, Belser UC. Clinical performance of novel-design porcelain veneers for the recovery of coronal volume and length. Int J Periodontics Restorative Dent 2000;20(5):440-457 
22 Shaini FJ, Shortall ACC, Marquis PM. Clinical performance of porcelain laminate veneers. A retrospective evaluation over a period of 6.5 years. J Oral Rehabil 1997;24(8):553-559

23 Fradeani M. Six-year follow-up with Empress veneers. Int J Periodontics Restorative Dent 1998;18(3):216-225

24 Aykor A, Ozel E. Five-year clinical evaluation of 300 teeth restored with porcelain laminate veneers using totaletch and a modified self-etch adhesive system. Oper Dent 2009;34(5):516-523

25 Guess PC, Stappert CF. Midterm results of a 5-year prospective clinical investigation of extended ceramic veneers. Dent Mater 2008;24(6):804-813

26 Murphy E, Ziada HM, Allen PF. Retrospective study on the performance of porcelain laminate veneers delivered by undergraduate dental students. Eur J Prosthodont Restor Dent 2005;13(1):38-43

27 Aristidis GA, Dimitra B. Five-year clinical performance of porcelain laminate veneers. Quintessence Int 2002;33(3):185-189

28 Peumans M, Van Meerbeek B, Lambrechts P, Vuylsteke-Wauters M, Vanherle G. Five-year clinical performance of porcelain veneers. Quintessence Int 1998;29(4):211-221

29 Gurel G, Sesma N, Calamita MA, Coachman C, Morimoto S. Influence of enamel preservation on failure rates of porcelain laminate veneers. Int J Periodontics Restorative Dent 2013;33(1):31-39

30 Granell-Ruiz M, Fons-Font A, Labaig-Rueda C, Martínez-González A, Román-Rodríguez JL, Solá-Ruiz MF. A clinical longitudinal study 323 porcelain laminate veneers. Period of study from 3 to 11 years. Med Oral Patol Oral Cir Bucal 2010;15(3):e531-e537

31 Dumfahrt H, Schäffer H. Porcelain laminate veneers. A retrospective evaluation after 1 to 10 years of service: Part IIClinical results. Int J Prosthodont 2000;13(1):9-18

32 Burke FJT, Lucarotti PSK. Ten-year outcome of porcelain laminate veneers placed within the general dental services in England and Wales. J Dent 2009;37(1):31-38
33 Peumans M, De Munck J, Fieuws S, Lambrechts P, Vanherle G, Van Meerbeek B. A prospective ten-year clinical trial of porcelain veneers. J Adhes Dent 2004;6(1):65-76

34 Friedman MJ. A 15-year review of porcelain veneer failure-a clinician's observations. Compend Contin Educ Dent 1998;19(6):625-628, 630, 632 passim, quiz 638

35 Layton D, Walton T. An up to 16-year prospective study of 304 porcelain veneers. Int J Prosthodont 2007;20(4):389-396

36 Layton DM, Walton TR. The up to 21-year clinical outcome and survival of feldspathic porcelain veneers: accounting for clustering. Int J Prosthodont 2012;25(6):604-612

37 Hoy D, Brooks P, Woolf A, et al. Assessing risk of bias in prevalence studies: modification of an existing tool and evidence of interrater agreement. J Clin Epidemiol 2012;65(9):934-939

38 Olley RC, Andiappan M, Frost PM. An up to 50-year follow-up of crown and veneer survival in a dental practice. J Prosthet Dent 2018;119(6):935-941

39 Beier US, Kapferer I, Burtscher D, Dumfahrt H. Clinical performance of porcelain laminate veneers for up to 20 years. Int J Prosthodont 2012;25(1):79-85

40 Guyatt G, Cairns J, Churchill D, et al; Evidence-Based Medicine Working Group. Evidence-based medicine. A new approach to teaching the practice of medicine. JAMA 1992;268(17):2420-2425

41 Slavin RE. Best-evidence synthesis: an alternative to meta-analytic and traditional reviews. Educ Res 1986;15(9):5-11

42 Moher D, Tetzlaff J, Tricco AC, Sampson M, Altman DG. Epidemiology and reporting characteristics of systematic reviews. PLoS Med 2007;4(3):e78

43 Harhash AY, ElSayad II, Zaghloul AGS. A comparative in vitro study on fluoride release and water sorption of different flowable esthetic restorative materials. Eur J Dent 2017;11(2):174-179

44 Linhares LA, Pottmaier LF, Lopes GC. Fracture resistance of veneers in premolars. Eur J Dent 2018;12(2):191-198

45 Lin DY, Wei LJ. The robust inference for the proportional hazards model. J Am Stat Assoc 1989;84:1074-1078 